\title{
Avoiding or Reversing Hartmann's Procedure Provides Improved Quality of Life After Perforated Diverticulitis
}

\author{
Jefrey Vermeulen • Martijn P. Gosselink • \\ Jan J. V. Busschbach • Johan F. Lange
}

Received: 25 September 2009 / Accepted: 4 January 2010/Published online: 2 February 2010

(C) 2010 The Author(s). This article is published with open access at Springerlink.com

\begin{abstract}
Introduction The existing literature regarding acute perforated diverticulitis only reports about short-term outcome; longterm following outcomes have not been assessed before. The aim of this study was to assess long-term quality of life (QOL) after emergency surgery for perforated diverticulitis.

Patients and Methods Validated QOL questionnaires (EQ-VAS, EQ-5D index, QLQ-C30, and QLQ-CR38) were sent to all eligible patients who had undergone emergency surgery for perforated diverticulitis in five teaching hospitals between 1990 and 2005. Differences were compared between patients that had undergone Hartmann's procedure (HP) or resection with primary anastomosis (PA) and also compared to a sex- and age-matched sample of healthy subjects.

Results Of a total of 340 patients, only 150 patients (44\%) were found still alive in July 2007 (median follow-up 71 months). The response rate was $87 \%$. In patients with PA, QOL was similar to the general population, whereas QOL after HP was significantly lower. The presence of a stoma was found to be an independent factor related to worse QOL. The deterioration in QOL was mainly due to problems in physical function and body image.

Conclusions Survivors after perforated diverticulitis had a worse QOL than the general population, which was mainly due to the presence of an end colostomy. QOL may improve if these stomas are reversed or not be performed in the first place.
\end{abstract}

Keywords Perforated diverticulitis · Quality of life ·

Hartmann's procedure $\cdot$ Primary anastomosis

Meeting presentation Annual Meeting of the Dutch Society of Surgery. Long-term quality of life after emergency surgery for perforated diverticulitis. 15th May 2009, Veldhoven, The Netherlands

J. Vermeulen $(\bowtie) \cdot J$. F. Lange

Department of Surgery, Erasmus University Hospital,

Dr. Molewaterplein 40,

3015 GD Rotterdam, The Netherlands

e-mail: j.vermeulen.1@erasmusmc.nl

M. P. Gosselink

Department of Surgery, Maasstad Hospital,

Groene Hilledijk 315,

3075 EA Rotterdam, The Netherlands

\section{J. J. V. Busschbach}

Department for Medical Psychology and Psychotherapy,

Erasmus University Rotterdam,

Dr. Molewaterplein 40,

3015 GD Rotterdam, The Netherlands

\section{Introduction}

Diverticulitis is one of the most common diseases related to the gastrointestinal tract in western countries. In The Netherlands, 14,000 new cases have been estimated for 2006, which equals an incidence of 80/100,000 patients each year. ${ }^{1}$ In spite of this, only $2 \%$ of these patients who present for urgent evaluation have acute perforation due to diverticulitis. ${ }^{2}$

Emergency surgery for perforated diverticulitis is associated with substantial morbidity and mortality, regardless of selected strategy. ${ }^{3}$ For many surgeons, Hartmann's procedure (HP) still remains the favored option, but it leaves the patient with an end colostomy. It is well known that patients with stomas may face both physical and psychological difficulties. ${ }^{4}$ Reversal of HP is also associated with substantial morbidity and even mortality. ${ }^{5}$ This is one of the main reasons why HP is never reversed in about half of patients.

Improvements in surgical and radiological intervention techniques and progress in the management of peritoneal 
sepsis have led to an increasing interest in colonic resection with primary anastomosis (PA). Although not proven in randomized controlled trials, PA seems not to be inferior to HP in terms of postoperative complications and mortality. ${ }^{3,}{ }^{6-8}$ In these patients, a stoma can be withheld or, in case of a defunctioning loop ileostomy, reversed easily and quickly. ${ }^{5}$

The existing literature regarding perforated diverticulitis only reports on short-term outcome such as mortality and postoperative complications. Patient-orientated outcomes, such as quality of life (QOL), have never been assessed previously in patients needing emergency surgery for acute perforated diverticulitis. Quality of life is increasingly recognized as a crucial factor when assessing clinical outcomes after surgical interventions as it presents a patient's perspective, which is obviously a key outcome in clinical decision-making. To provide more data from a patient's perspective, the present study evaluates the long-term functional and health-related QOL outcomes of patients after emergency surgery for acute perforated diverticulitis. QOL will be examined in relation to surgical technique (HP or PA), surgeon's experience in colorectal surgery, severity of the primary disease, and patients' characteristics.

\section{Patients and Methods}

A cohort of 340 consecutive patients had undergone emergency surgery for perforated diverticulitis between January 1990 and December 2005 at the surgical departments of the academic and the four major teaching hospitals of Rotterdam, The Netherlands (Erasmus University Medical Centre, Ikazia Hospital, Medical Centre Rijnmond-Zuid (formerly St. Clara Hospital and Zuider Hospital), and St. Franciscus Gasthuis Hospital). Patients were selected from computerized surgery registration databases using the search codes: exploratory laparotomy, diverticulitis, perforated hollow viscus, Hartmann's procedure, left hemicolectomy, sigmoid resection, low anterior resection. Using this searching strategy, a complete overview of all patients presenting at the surgical units of the hospitals mentioned above was obtained, as all patients with acute perforated diverticulitis undergo emergency surgery. The indications for surgery were clinical signs of diffuse peritonitis or presence of septic status with acute abdominal pain, free gas on plain abdominal radiography, or specific findings for perforated diverticulitis at ultrasonography or computerized tomography (CT). The type of surgical procedure (HP $n=238$; PA $n=93$; suture repair $n=9$ ) was left to the discretion of the surgeon on call. There were no laparoscopic emergency operations performed during the study period.

Follow-up of all patients was conducted until July 2007. Data regarding the patients' course after initial hospital discharge was gathered from the hospitals' medical reports and by telephone inquiry from the patients themselves or when necessary, the patient's direct relatives and general practitioner. At 1 July 2007, 150 patients were still alive (HP $n=90$; PA $n=58$; suture repair $n=3$ ). Ten patients were lost in follow-up as they moved abroad $(n=3)$ or their home addresses were not available $(n=7)$. Validated questionnaires were sent by post to all 150 eligible patients after they were asked by phone to participate. The response rate was $87 \%$ (131 patients).

In order to assess long-term QOL, the patients filled in the EuroQol EQ-VAS, the EQ-5D index, and the European Organization for Research and Treatment of Cancer (EORTC) QLQ-C30 and QLQ-CR38 questionnaires. The EQ-VAS is a single-item visual analog scale (VAS), ranging from 0 "worse imaginable health state" to 100 "best imaginable health state." The EQ-VAS represents the "value" of the current health state from a patient perspective. Next to the EQ-VAS, the patients classify their current health state using the five items of the EuroQol EQ-5D. This classification can be transformed to a so-called index score representing "the societal value" of the health state. Such societal value represents the value the general public attaches to current health state of the patient. ${ }^{9}$

Disease-specific QOL was measured according to the official scoring procedures for the EORTC QLQ-C30 and EORTC QLQ-CR38 questionnaires. Both were originally developed to assess the QOL of cancer patients from a patient's perspective. ${ }^{11}$ Other than the EuroQol, the outcomes of the EQRTC questionnaires are multidimensional. EORTC QLQ-C30 contains 30 items that can be computed in five functional scales (physical, role, emotional, cognitive, and social functioning), three symptom scales, and six single items (fatigue, nausea and vomiting, pain, dyspnea, insomnia, loss of appetite, constipation, diarrhea, and financial difficulties). ${ }^{12}$

The EORTC QLQ-CR38 is subdivided into two functional scales (i.e., body image and sexual functioning), seven symptom scales (micturition problems, gastrointestinal tract symptoms, chemotherapy side effects, defecation problems, stoma-related problems, and male and female sexual problems), and three single-item measures (sexual enjoyment, weight loss, and future perspective).

The validity and reliability of both the EORTC QLQ C30 and QLQ-CR38 have been established in Dutch patients with colorectal cancer. ${ }^{11}$ In both questionnaires, scores are summed within scales and rescaled from 0 to 100. A higher score indicates better functioning, future perspective, and a lower level of symptomatology.

Differences in QOL were determined and compared between patients that underwent HP or PA. Both groups were also compared to a sex- and age-matched communitybased sample of healthy people in The Netherlands. ${ }^{10}$ 
Categorical variables were compared using the Chi-square test or Fisher's exact test. Continuous variables were compared using the Mann-Whitney test. Differences in QOL between the two surgical groups were determined with multivariate logistic regression analysis, adjusting for age, gender, American Society of Anesthesiologist (ASA) classification, Hinchey score, and the presence of a stoma. These variables were a priori hypothesized as potential confounders based on literature and/or significant clinical variables in univariate analysis. Differences were considered statistically significant at a two-tailed $p$ value of $<0.05$.

\section{Results}

Patient characteristics regarding type of procedure and clinical data are listed in Table 1. Responders were similar to the nonresponders regarding gender, age, and surgical procedure. Patients that had undergone HP had significant higher ASA and Hinchey scores during primary surgery compared to patients that underwent PA $(p<0.01$ and $p=0.04$, respectively). PA was more frequently performed by specialist colorectal surgeons $(p=0.03)$. At time of the questionnaire, $30 \mathrm{HP}$ patients (39\%) still had an end colostomy. Two PA patients still had a loop ileostomy (4\%). The median duration of time interval between the operation and the questionnaire was 71 months (range 23-205 months).

The mean scores and ranges of the EQ-VAS and EQ-5D index are presented in Table 2. From the patient perspective, the mean general QOL score (EQ-VAS) was better after PA compared to HP. Also from the social perspective, the mean
EQ-5D index score was better in patients after PA. In patients who had undergone PA, the EQ-VAS and EQ-5D index score was similar to that of the general population. The patients who had undergone HP had a significantly lower EQ-VAS and EQ-5D scores compared to the sex-age-matched general population (EQ-VAS $p<0.01$; EQ-5D $p=0.02$ ).

The presence of a stoma was found to be an independent factor related to the QOL, with patients without a stoma having a better QOL (EQ-VAS $p=0.03$; EQ-5D $p=0.04$ ). When assessing a subgroup containing patients after HP who had undergone restoration of bowel continuity $(n=46)$, differences in QOL from the patients perspective and the social perspective were no longer significant compared to the general population and the patients after PA.

No difference in QOL was found between patients who were operated by an experienced colorectal surgeon or a general surgeon. There was no correlation between Hinchey scores and QOL scores. Higher ASA classifications were associated with a lower QOL (EQ-VAS $p=0.04$; EQ-5D $p=0.01)$. ASA classification and type of surgery were significantly related to QOL in bivariate analyses but were not found to be significant in multivariate analysis. Only the presence of a colostomy was found to be an independent predictor for lower QOL (EQ-VAS odds ratio 2.4; $95 \%$ CI 1.2 to $4.8 ; p=0.03$ ) after multivariate logistic regression analysis.

Scores of the EORTC QLQ-CR30 and the QLQ-CR38 for the patient groups are presented in Tables 3 and 4 . Differences between HP and PA were found on five scales. PA patients had significantly higher scores with regard to global health status, physical function, fatigue, dyspnea,

Table 1 Baseline Characteristics of the Responders

\begin{tabular}{llll}
\hline & Primary Surgery & & Suture repair \\
\cline { 2 - 4 } & HP & PA & 2 \\
\hline Numbers of patients & 76 & 53 & 56 \\
Age & 62 & 59 & 134 \\
Length of follow-up (months) & 72 & 69 & $50 / 50$ \\
Male/female (\%) & $52 / 48$ & $40 / 60$ & $0(0 \%)$ \\
Patients with stoma ${ }^{\mathrm{a}}$ & $30(39 \%)$ & $2(4 \%)$ & $100 / 0 / 0 / 0$ \\
ASA I/II/III/IV (\%) & a & $41 / 34 / 17 / 8$ & $50 / 0 / 50 / 0$ \\
Hinchey I/II/III/IV (\%) ${ }^{\mathrm{a}}$ & $25 / 28 / 33 / 14$ & $23 / 43 / 26 / 8$ & $100 / 0$ \\
MPI $<$ 26/MPI $=26$ & $24 / 12 / 52 / 12$ & $86 / 14$ & 100 \\
Reintervention (\%) & $93 / 7$ & 13 & $50 / 50$ \\
Specialist colorectal surgeon & & \\
\hline
\end{tabular}

Data are median numbers with percentages in parentheses, unless otherwise specified. $\mathrm{HP}=$ Hartmann procedure, $P=$ primary anastomosis., $\mathrm{ASA}=$ American Society of Anaesthesiologist classification, MPI=Mannheim peritonitis index.

$\mathrm{a}=\mathrm{PA}$ vs. HP: $\mathrm{P}<0.05 ; \mathrm{b}=$ Reinterventions were defined as radiological-assisted percutaneous drainage of abdominal or pelvic abscess, open abdominal wound management or reoperation for ongoing sepsis, abdominal abscess, evisceration, anastomotic leakage or stoma-related complications. 
Table 2 General Quality of Life Scores

\begin{tabular}{llll}
\hline & HP & PA & Population \\
\hline EQ-VAS & $65(20-100)$ & $74(10-100)^{\mathrm{a}}$ & $79(68-87)^{\mathrm{b}}$ \\
EQ-5D index & $67(-18-100)$ & $77(67-93)^{\mathrm{a}}$ & $77(67-92)^{\mathrm{b}}$ \\
\hline
\end{tabular}

Data are mean scores with ranges in parentheses. EQ-VAS=Quality of life from the patient perspective, EQ-5D index=Quality of life from the social perspective. $\mathrm{HP}=$ Hartmann procedure, $\mathrm{PA}=$ primary anastomosis. Population $=\mathrm{a}$ sex- and age-matched community-based sample of healthy Dutch persons.

$\mathrm{a}=$ PA vs HP: $\mathrm{p}<0.05 ; \mathrm{b}=$ Population vs. HP: $\mathrm{p}<0.05$.

and body image compared to HP patients. The QOL from the patient perspective (EQ-VAS) was affected by the presence of physical function problems and body image problems $(p<0.01$ and $p=0.04$, respectively). Global health status, physical function, fatigue, and body image were predictors of QOL in social perspective (EQ-5D) (global health status, physical function, and fatigue, all $p<0.01$; body image $p=0.04$ ). Again, after assessing the patients who had undergone HP followed by restoration of bowel continuity, the EORTC QLQ-CR30 and QLQ-CR38 scores were not significantly different compared to those of patients that had undergone PA.

\section{Discussion}

The present study compared long-term QOL among patients that underwent HP and PA for acute perforated diverticulitis. The QOL outcomes were also compared to the general Dutch population. Survivors from acute perforated diverticulitis reported worse QOL compared to the Dutch population. QOL in patients who had undergone HP was lower compared to patients who underwent PA, both from the patient's and a social perspective. After reversal of HP, this difference disappeared, but HP reversal was performed in only $61 \%$ of the patients. QOL in patients after perforated diverticulitis was mainly influenced by the presence of a stoma postoperatively.

Functional aspects and QOL of survivors have become increasingly important for patients with perforated diverticulitis because survival after emergency surgery for perforated diverticulitis is poor, both in the short and long terms. ${ }^{13}$ Previously, we described the long-term survival after perforated diverticulitis. ${ }^{13}$ The 5 -year mortality after perforated diverticulitis was approximately $50 \%$. The main reason for this observation was the poor general condition of the patients. Almost half of the patients that presented with acute perforated diverticulitis were classified ASA III or IV, and one third was older than 75 years of age. ${ }^{13}$ It is stated before that the incidence of perforated diverticulitis is highest in elderly patients, suffering from multiple comorbidities as indicated by a higher ASA classification. ${ }^{14}$ These factors are known to be correlated with mortality. Direct postoperative mortality after perforated diverticulitis can increase to $40 \%$ in patients of older age. ${ }^{15}$ It is therefore not surprising that this group of patients have a poor prognosis in the short and long term.

QOL of patients is also important for surgeons when making a decision about the strategy of primary surgery. The optimal surgical treatment of perforated diverticulitis is still a matter of debate; neither PA nor HP has been proven the superior surgical strategy in terms of mortality and
Table 3 Disease Specific Quality of Life Scores (EORTC QLQ-C30)

A high subscale score indicates low distress and good functioning. $\mathrm{HP}=$ Hartmann procedure, $\mathrm{PA}=$ primary anastomosis. $\mathrm{a}=$ PA vs HP: $\mathrm{p}<0.05$.

\begin{tabular}{|c|c|c|c|c|}
\hline & \multicolumn{2}{|l|}{ HP } & \multicolumn{2}{|l|}{ PA } \\
\hline & Mean & Median (range) & Mean & Median (range) \\
\hline Physical function ${ }^{a}$ & 66 & $67(0-100)$ & 79 & $87(13-100)$ \\
\hline Role function & 70 & $92(0-100)$ & 76 & $100(17-100)$ \\
\hline Emotional function & 77 & $92(0-100)$ & 81 & $92(0-100)$ \\
\hline Cognitive function & 78 & $83(17-100)$ & 85 & $100(17-100)$ \\
\hline Social function & 75 & $83(0-100)$ & 76 & $89(0-100)$ \\
\hline Global health status $^{\text {a }}$ & 69 & $71(14-100)$ & 79 & $86(14-100)$ \\
\hline Fatigue $^{\mathrm{a}}$ & 64 & $67(0-100)$ & 76 & $89(0-100)$ \\
\hline Nausea/vomiting & 92 & $100(33-100)$ & 94 & $100(50-100)$ \\
\hline Pain & 74 & $100(0-100)$ & 79 & $100(0-100)$ \\
\hline Dyspnoea $^{\text {a }}$ & 65 & $67(0-100)$ & 80 & $100(0-100)$ \\
\hline Sleep disturbance & 70 & $100(0-100)$ & 67 & $67(0-100)$ \\
\hline Appetite loss & 85 & $100(0-100)$ & 85 & $100(0-100)$ \\
\hline Constipation & 86 & $100(0-100)$ & 77 & $100(0-100)$ \\
\hline Diarrhea & 87 & $100(0-100)$ & 87 & $100(0-100)$ \\
\hline Financial worries & 85 & $100(0-100)$ & 91 & $100(0-100)$ \\
\hline
\end{tabular}


Table 4 Disease Specific Quality of Life Scores (EORTC QLQ-CR38)
A high subscale score indicates low distress and good functioning. $\mathrm{HP}=$ Hartmann procedure, $\mathrm{PA}=$ primary anastomosis. $\mathrm{a}=$ PA vs HP: $\mathrm{p}<0.05$.

\begin{tabular}{|c|c|c|c|c|}
\hline & \multicolumn{2}{|l|}{ HP } & \multicolumn{2}{|l|}{ PA } \\
\hline & Mean & Median (range) & Mean & Median (range) \\
\hline Micturition problems & 75 & $78(0-100)$ & 78 & $78(22-100)$ \\
\hline Gastrointestinal problems & 82 & $87(13-100)$ & 81 & $87(13-100)$ \\
\hline Weight loss & 86 & $100(33-100)$ & 93 & $100(33-100)$ \\
\hline Body image ${ }^{a}$ & 72 & $85(0-100)$ & 80 & $89(0-100)$ \\
\hline Defaecation problems & 86 & $90(43-100)$ & 89 & $95(48-100)$ \\
\hline Stoma problems & 73 & $76(0-100)$ & 81 & $81(81-81)$ \\
\hline Chemo side effects & 82 & $89(33-100)$ & 83 & $100(33-100)$ \\
\hline Sexual function & 21 & $17(0-67)$ & 20 & $17(0-67)$ \\
\hline Sexual enjoyment & 48 & $33(0-100)$ & 58 & $67(0-100)$ \\
\hline Male sex problems & 61 & $67(0-100)$ & 69 & $100(0-100)$ \\
\hline Female sex problems & 86 & $100(17-100)$ & 81 & $83(33-100)$ \\
\hline Future perspective & 64 & $67(0-100)$ & 74 & $67(0-100)$ \\
\hline
\end{tabular}

morbidity. Because the impairment of function that may occur after different operations varies considerably, an assessment of QOL for each type of surgical procedure is becoming an essential principle to follow in a successful healthcare system. ${ }^{16}$ A very small number of studies have evaluated QOL in patients with (complicated) diverticulitis, but the literature is diverse and frequently based on heterogeneous pathologies. The present study is the first to assess patient's long-term QOL after emergency surgery for acute perforated diverticulitis.

One study used questionnaires to examine whether diverticular disease has an impact on QOL. The authors suggested that the lower QOL scores found in diverticulitis patients compared to healthy controls could be useful in decision making and selection of patients for elective surgical treatment. ${ }^{17}$ Two studies assessed long-term QOL after laparoscopic or open sigmoid resection for uncomplicated diverticulitis. Both found no significant differences between the two surgical techniques. ${ }^{18,19}$ A recent study comparing two surgical strategies for treatment of complicated diverticular disease showed no difference in QOL in patients after PA or HP. ${ }^{20}$ Unfortunately, this study was performed in a heterogeneous group of patients varying from emergency surgery for diverticular peritonitis to elective laparoscopic surgery in patients failing conservative treatment of diverticulitis. A recent study of qualityadjusted life-years after surgery for diverticular peritonitis concluded that PA was superior to HP, unless the risk of postoperative complication was higher than $40 \%{ }^{21}$ The conclusions were based on decision analysis techniques for a hypothetical 65-year old, with the use of so-called utilities (a measure of the patient's or surgeon's relative preference for each individual outcome) to calculate. Unfortunately, there are no available published utilities specifically for diverticular disease, and therefore, many of the used utilities arose from studies assessing other colorectal diseases, small or dated studies, expert judgment, and assumptions. Given the state of imperfect evidence and the high degree of uncertainty, the conclusion that PA was superior to HP in terms of quality-adjusted life expectancy is at least doubtful.

This study, however, included only patients with acute perforated diverticulitis and supports the conclusion that PA is superior to HP in terms of QOL. The indications for surgery were clinical signs of diffuse peritonitis or presence of septic status with acute abdominal pain or specific findings for perforated diverticulitis at radiographic investigations. The decision for surgical management was made by the surgeon on call, and not all patients had undergone preoperative CT-scanning. Therefore, some patients showed Hinchey I or II perforated diverticulitis at surgery. It is now recognized that patients with small, contained perforations, who are not systemically ill can be treated initially with antibiotics alone or by CT-guided percutaneous drainage. ${ }^{22}$ In this study, all patients that underwent emergency surgery for acute perforated diverticulitis were examined. Patients underwent either HP or resection with PA.

QOL in patients who underwent HP and PA were compared after a median of 71 months after primary surgery using the EQ-VAS, EQ-5D, and QLQ-C30 and QLQ-CR38. These questionnaires have proven to be efficient and reliable tools for establishing the health status within the Dutch community. ${ }^{12,23}$ General QOL scores were significantly lower in patients that had undergone HP compared to patients after PA. HP patients were associated with lower physical performances mainly due to a lower physical function and more complains of fatigue. They also showed a diminished body image compared to PA patients.

QOL of patient after PA was comparable with the general population both from the patient's and societal 
perspective. Although not significant, QOL from the patient's perspective (EQ-VAS, Table 2) in PA patients was lower than the general Dutch population. This might not be so much a difference in perspective as it is a difference in the methods: the societal perspective of the EQ-5D is based on "time trade off" (TTO), and the patient perspective is based on a VAS. TTO is a more conservative valuation of burden of disease than VAS. ${ }^{24}$ Furthermore, the EQ-5D societal perspective is known to produce a ceiling effect. ${ }^{25}$ This could explain why EQ-VAS remained significantly higher in PA patients than HP patients, whereas EQ-5D did not, after multivariate analysis.

Patients that had undergone HP and subsequent reversal of their end colostomy in a second operation showed comparable QOL outcomes to patients that had undergone PA and the general population. HP reversal was performed in only $61 \%$ of the patients, which is in reflective of the literature. ${ }^{26}$ It has been stated previously that patients with a stoma may face many difficulties both physical and psychological. ${ }^{4}$ It is known that patients with direct intestinal continuity after surgery for colon cancer showed better QOL scores than those who received an end colostomy. ${ }^{27}$ Also, when having a stoma, reversal of it can result in significant improvements in global QOL and physical and social function. ${ }^{28}$ In the present study, patients without an end colostomy (PA) and the HP patients who had their stoma reversed showed similar QOL scores from a social perspective to the general population, whereas patients with an end colostomy (HP without reversal) showed a worse QOL. QOL was independently related to body image problems, e.g., the presence of a stoma.

In the light of body image problems, the use of minimally invasive treatment strategies for perforated diverticulitis may be an interesting development. Although its exact role is still unclear, several studies have shown excellent results in treatment of patients with peritonitis due to perforated diverticulitis by laparoscopic peritoneal lavage and drainage. ${ }^{29-31}$ Compared to HP or PA, laparoscopic lavage and drainage seems to have a lower morbidity and mortality rate. And in most patients a subsequent elective resection is probably unnecessary so a stoma can be avoided. ${ }^{30,31}$ As the presence of a stoma plays an important role in QOL in the patients after perforated diverticulitis, laparoscopic procedures might not only appear to be superior over HP and PA in the short term (postoperative morbidity and mortality) but also in the long-term (QOL). Future comparative studies must confirm these statements.

Although QOL was mainly affected by body image problems, it is also important when interpreting the QOL scores to consider that the outcomes are dependent on patient's preoperative expectations. Fear or ignorance of the long-term consequences of the operation is associated with lower QOL. ${ }^{32}$ Optimistic and well-informed patients may be more resistant to the negative influence of limitations (caused by their stoma) on their QOL. Surgeons, with support from the stoma care therapist and the multidisciplinary team, can anticipate this by offering appropriate education regarding a colostomy. Ideally, education should start preoperatively so patients require less time and have fewer problems with their rehabilitation. ${ }^{33}$ When a colostomy becomes necessary, modern stoma appliances are so effective that most patients with a colostomy can enjoy normal lives. Engel et al. who reported that patients after an abdominal perineal resection have a consistently lower quality of life stated that $60 \%$ of the patients in their sample were poorly informed about stoma irrigation techniques. ${ }^{34}$ This underlines the importance of instruction and education by stoma care therapists on colostomy care and washout, enabling more bowel control. In patients with acute perforated diverticulitis, preoperative counseling is not possible, as these patients require immediate surgery. Postoperative counseling of the patient (and family) is therefore very important to obtain better control of their bowel function and hence improve long-term outcome.

General health prior to surgery is also associated with postoperative QOL. In general, patients with more than one comorbid condition report the poorest level of QOL. ${ }^{35}$ It is known, for example, that neurological comorbidities, like cerebral vascular accident or multiple sclerosis, may be a reason for stoma care problems, causing lower quality of life. ${ }^{36}$ Patients with higher ASA classifications (multiple comorbidities) experience more functional limitations and show a lower QOL life than ASA I patients. ${ }^{32}$ In this study, patients who underwent HP had significant higher ASA classifications and Hinchey scores prior to initial surgery compared to patients after PA. Therefore, it seems that the more severely affected and high-risk patients underwent HP. These patients appeared to have lower postoperative QOL scores than patients after PA and the general Dutch population. To assess whether the lower QOL was caused by differences in patient's characteristics and severity of disease scores (selection bias), a multivariate analysis was performed. After adjusting for the differences between the groups, QOL remained worse after HP compared to PA.

In conclusion, survivors after perforated diverticulitis had a worse QOL compared to the general Dutch population, which is mainly related to the presence of an end colostomy. When such stoma can be avoided (PA) or reversed, the QOL in these patients may improve.

Acknowledgments We thank E. van der Harst and P.P.L.O. Coene of the Maasstad Hospital, W.F. Weidema of the Ikazia Hospital and G. H.H. Mannaerts of the St. Franciscus Gasthuis, all in Rotterdam, The Netherlands and J.C. Oliver of the Groote Schuur Hospital in Cape Town, South Africa for their contributions in the production of this manuscript. 
Open Access This article is distributed under the terms of the Creative Commons Attribution Noncommercial License which permits any noncommercial use, distribution, and reproduction in any medium, provided the original author(s) and source are credited.

\section{References}

1. National Medical Registration (The Netherlands), Prismant. www. prismant.nl

2. Jacobs DO. Clinical practice. Diverticulitis. N Engl J Med. 2007 15;357:2057-2066

3. Vermeulen J, Akkersdijk GP, Gosselink MP, Hop WC, Mannaerts GH, van der Harst E, Lange JF, et al. Outcome after emergency surgery for acute perforated diverticulitis in 200 cases. Dig Surg 2007;24:361-6

4. Nugent KP, Daniels P, Stewart B, Patankar R, Johnson CD. Quality of life in stoma patients. Dis Colon Rectum 1999;42:1569-1574

5. Vermeulen J, Coene PPLO, Hout NM van, Harst E van der, Mannaerts GHH, Weidema WF, Lange JF. Restoration of bowel continuity after surgery for acute perforated diverticulitis: should Hartmann's procedure be considered a one-stage procedure? Colorectal Dis 2009;11:619-624

6. Salem L, Flum DR. Primary anastomosis or Hartmann's procedure for patients with diverticular peritonitis? A systematic review. Dis Colon Rectum 2004;47:1953-1964.

7. Gooszen AW, Tollenaar RA, Geelkerken RH, Smeets HJ, Bemelman WA, Van Schaardenburgh P, et al. Prospective study of primary anastomosis following sigmoid resection for suspected acute complicated diverticular disease. Br J Surg 2001;88:693-697.

8. Schilling MK, Maurer CA, Kollmar O, Buchler MW. Primary vs. secondary anastomosis after sigmoid colon resection for perforated diverticulitis (Hinchey Stage III and IV): a prospective outcome and cost analysis. Dis Colon Rectum 2001;44: 699-703

9. Lamers LM, McDonnell J, Stalmeier PF, Krabbe PF, Busschbach JJ. The Dutch tariff: results and arguments for an effective design for national EQ-5D valuation studies. Health Econ 2006;15:1121-1132

10. Stolk EA, Busschbach JJV, Krabbe PFM, Using the Internet to collect EQ-5D norm scores: a valid alternative? Accepted for publication in the Proceedings of the Plenary Meeting of the EuroQoL Group, The Hague 2007 (www.euroqol.org)

11. Sprangers MA, te Velde A, Aaronson NK. The construction and testing of the EORTC colorectal cancer-specific quality of life questionnaire module (QLQ-CR38). European Organization for Research and Treatment of Cancer Study Group on Quality of Life. Eur J Cancer 1999;35:238-247

12. Aaronson NK, Ahmedzai S, Bergman B, Bullinger M, Cull A, Duez NJ, Filiberti A, Flechtner H, Fleishman SB, de Haes JC, et al. The European Organization for Research and Treatment of Cancer QLQ-C30: a quality-of-life instrument for use in international clinical trials in oncology. J Natl Cancer Inst 1993;85:365-376

13. Vermeulen J, Gosselink MP, Hop WCJ, Van der Harst E, Hansen BE, Mannaerts GHH, Coene PPLO, Weidema WF, Lange JF. Long-term survival after perforated diverticulitis. Colorectal Dis 2009 Nov 6. [Epub ahead of print]

14. 14 Morris CR et al. Incidence of perforated diverticulitis and risk factors for death in a UK population. Br J Surg. 2008;95:876-81

15. Pisanu A et al. Surgical treatment of perforated diverticular disease: evaluation of factors predicting prognosis in the elderly. Int Surg 2004; 89: 35-8

16. Sajid MS, Rimpel J, Iftikhar M, Baig MK. Use of health related quality of life tools in colorectal surgery. Acta Chir Belg 2007;107:623-9
17. Bolster LT, Papagrigoriadis S. Diverticular disease has an impact on quality of life - results of a preliminary study. Colorectal Dis 2003;5:320-3

18. Roblick UJ, Massmann A, Schwandner O, Sterk P, Krug F, Bruch HP, Schiedeck TH. Quality of life assessment after surgery for diverticulitis - a follow-up study (german). Zentralbl Chir 2002;127:31-5

19. Thaler K, Dinnewitzer A, Mascha E, Arrigain S, Weiss EG, Nogueras JJ, Wexner SD. Long-term outcome and health-related quality of life after laparoscopic and open colectomy for benign disease. Surg Endosc 2003; 17:1404-8

20. Constantinides VA, Aydin HN, Tekkis PP, Fazio VW, Heriot AG, Remzi FH. Long-term, health-related, quality of life comparison in patients undergoing single stage vs staged resection for complicated diverticular disease. Colorectal Dis 2006;8:663-71

21. Constantinides VA, Heriot A, Remzi F, Darzi A, Senapati A, Fazio VW, Tekkis PP. Operative strategies for diverticular peritonitis: a decision analysis between primary resection and anastomosis versus Hartmann's procedures. Ann Surg 2007;245:94-103

22. Cheadle WG, David A. Spain DA. The continuing challenge of intra-abdominal infection. Am J Surg 2003;186:15-22

23. Hoeymans N, van Lindert H, Westert GP. The health status of the Dutch population as assessed by the EQ-6D. Qual Life Res. 2005;14:655-63

24. Brooks R, Rabin R, Charro Fd. The Measurement and Valuation of Health Status Using EQ-5D: A European Perspective. 2003:143-65. ISBN 1-4020-1214-4

25. Brazier J, Roberts J, Tsuchiya A, Busschbach J. A comparison of the EQ-5D and SF-6D across seven patient groups. Health Economics 2004;13:873-84

26. Maggard MA, Zingmond D, O'Connell JB, Ko CY. What proportion of patients with an ostomy (for diverticulitis) get reversed? Am Surg 2004;70:928-31

27. Hassan I, Larson DW, Cima RR, Gaw JU, Chua HK, Hahnloser D, Stulak JM, O'Byrne MM, Larson DR, Wolff BG, Pemberton JH. Long-term functional and quality of life outcomes after coloanal anastomosis for distal rectal cancer. Dis Colon Rectum 2006; 49:1266-74

28. Camilleri-Brennan J, Steele RJ. Prospective analysis of quality of life after reversal of a defunctioning loop ileostomy. Colorectal Dis 2002;4:167-71

29. Franklin ME Jr, Portillo G, Treviño JM, Gonzalez JJ, Glass JL. Long-term experience with the laparoscopic approach to perforated diverticulitis plus generalized peritonitis. World J Surg. 2008;32:1507-11

30. Myers E, Hurley M, O'Sullivan GC, Kavanagh D, Wilson I, Winter DC. Laparoscopic peritoneal lavage for generalized peritonitis due to perforated diverticulitis. Br J Surg. 2008;95:97-101

31. Favuzza J, Friel JC, Kelly JJ, Perugini R, Counihan TC. Benefits of laparoscopic peritoneal lavage for complicated sigmoid diverticulitis. Int J Colorectal Dis 2009;24:797-801

32. Peters ML, Sommer M, de Rijke JM, Kessels F, Heineman E, Patijn J, Marcus MA, Vlaeyen JW, van Kleef M. Somatic and psychologic predictors of long-term unfavorable outcome after surgical intervention. Ann Surg 2007;245:487-94

33. Watson PG. The effects of short-term post-operative counselling on cancer/ostomy patients. Cancer Nurs 1983;6:21-9

34. Engel J, Kerr J, Schlesinger-Raab A, Eckel R, Sauer H, Hölzel D. Quality of life in rectal cancer patients: a four-year prospective study. Ann Surg 2003;238:203-13

35. Fortin M, Bravo G, Hudon C, Lapointe L, Almirall J, Dubois MF, Vanasse A. Relationship between multimorbidity and health-related quality of life of patients in primary care. Qual Life Res 2006;15:83-91

36. King RB. Quality of life after stroke. Stroke 1996;27:1467-72 